\title{
DEVELOPMENT OF STANDARD-FREQUENCY TRANSMITTING SETS
}

\author{
By L. Mickey and A. D. Martin
}

\section{ABSTRACT}

This paper describes the development of radio transmitting equipment used in the standard-frequency service provided by the Bureau since 1923. Difficulties encountered in the design and operation are discussed and improvements noted. In the establishment of the $5,000 \mathrm{kc} / \mathrm{s}$ schedules, innovations in frequency multiplication and control, based on the principle of the synchronized oscillator and harmonic amplifier, are described. Expansion of the transmitting equipment to the present $30-\mathrm{kw}$ installation is covered in detail. Diagrams and photographs of the Beltsville, Md., transmitting station are given.

\section{CONTENTS}

I. Introduction

II. Early standard-frequency transmitters

III. Changing demands of the service

IV. Choice of frequency

V. Synchronized oscillators.

VI. Harmonic amplifiers

VII. 30-kw transmitter

VIII. Results

\section{INTRODUCTION}

The widespread development of radio has brought increasing demand for close regulation of all radio frequencies to insure the maximum utilization of the available spectrum. In order to comply with governmental regulations, which become more and more exacting, each radio station must have access in some form to an accurate standard of frequency. This may be an independent standard of high accuracy, a substandard periodically compared by wire or radio with a precision standard, or a frequency supplied continuously by radio or wire from a precision standard.

National standards of frequency are maintained by the government laboratories of the various nations as bases of frequency assignment and administration. Comparisons with the national frequency standards are accomplished through three principal activities of the government laboratories: (1) Measurement of station frequencies; (2) measurement and calibration of frequency meters, piezoelectric oscillators, and other standards sent to the laboratory for that purpose, and (3) the radio transmission of standard-frequency signals. The first method becomes a large and expensive undertaking while the second is subject to inaccuracies resulting from changes of instruments in transit. Consequently, the third method of comparison has become increasingly important. It has proved to be the most accurate, economical, and practical means of dissemination of the national frequency standard. 
A radio transmission of standard frequency may consist of: (a) A standard radio-frequency signal, (b) a radio-frequency carrier modulated by a standard audio frequency, or $(c)$ a standard radio-frequency carrier modulated by a standard audio frequency. In Europe, the Laboratoire National de Radioelectricité and the National Physical Laboratory use a standard frequency of $1 \mathrm{kc} / \mathrm{s}$ to modulate a carrier frequency of approximately $200 \mathrm{kc} / \mathrm{s}$. As in (b) above, the accuracy of the carrier frequency is not held to be of major importance. The practice in the United States has been the transmission of unmodulated standard radio-frequency signals, although experimental transmissions of the $(c)$ classification are being conducted. Since early in 1923 the Bureau of Standards has provided a service of transmitting standard-frequency signals which at various times have covered the range from 125 to $10,000 \mathrm{kc} / \mathrm{s}$.

\section{EARLY STANDARD-FREQUENCY TRANSMITTERS}

The first apparatus used for the transmission of standard frequencies consisted of a single self-excited triode oscillator coupled to a simple antenna. It was adjusted to the desired frequency by means of a precision frequency meter. A stage of power amplification was later added for the purpose of minimizing the effects of varying antenna characteristics as well as increasing the distance range of transmission.

The development of the piezoelectric frequency standard provided a great improvement in the accuracy of the transmissions. Schedules were arranged so that all transmitted frequencies were multiples of a $100-$ or $200-\mathrm{kc} / \mathrm{s}$ standard. The oscillator was adjusted to the desired frequency by the method of beats between the transmitter and harmonics of the standard. A fine adjustment of the circuit capacitance made possible an accurate frequency setting.

Two transmitters were employed to cover approximately 30 frequencies between 125 and $6,000 \mathrm{kc} / \mathrm{s}$. Numerous difficulties limited the accuracy attainable. Poor regulation of power supply and temperature changes in tube and circuit elements caused some shifting of frequency. Furthermore, the wide range of frequencies made difficult the neutralization of the power amplifier.

Greater need for the service on the higher frequencies and the impracticability of transmissions within the broadcast band because of interference from the numerous stations of greater power, resulted in a shifting of the schedule to the range from 1,500 to $10,000 \mathrm{kc} / \mathrm{s}$. In 1930 one transmitter was dismantled and the other reconstructed for more efficient operation on the higher frequencies. An improved type of oscillator tube ana a 500-watt screen-grid power amplifier tube were installed. Oscillator inductors of heavy copper tubing were constructed, forced ventilation was applied, and an adequate power supply and filter system were provided. A variable-length, singlewire, voltage-fed antenna was employed. The length was adjusted to the optimum value for each operating frequency. A view of this transmitter is shown in figure 1.

While this equipment showed much improvement over the earlier, several limitations remained. Temperature changes in the oscillator, with the consequent frequency variations, persisted in spite of the improved ventilation. These were caused mainly by the large radiofrequency currents and the use of changeable inductors. In this connection many desirable changes suggested themselves. These 
B.S. Journal of Research, RP630

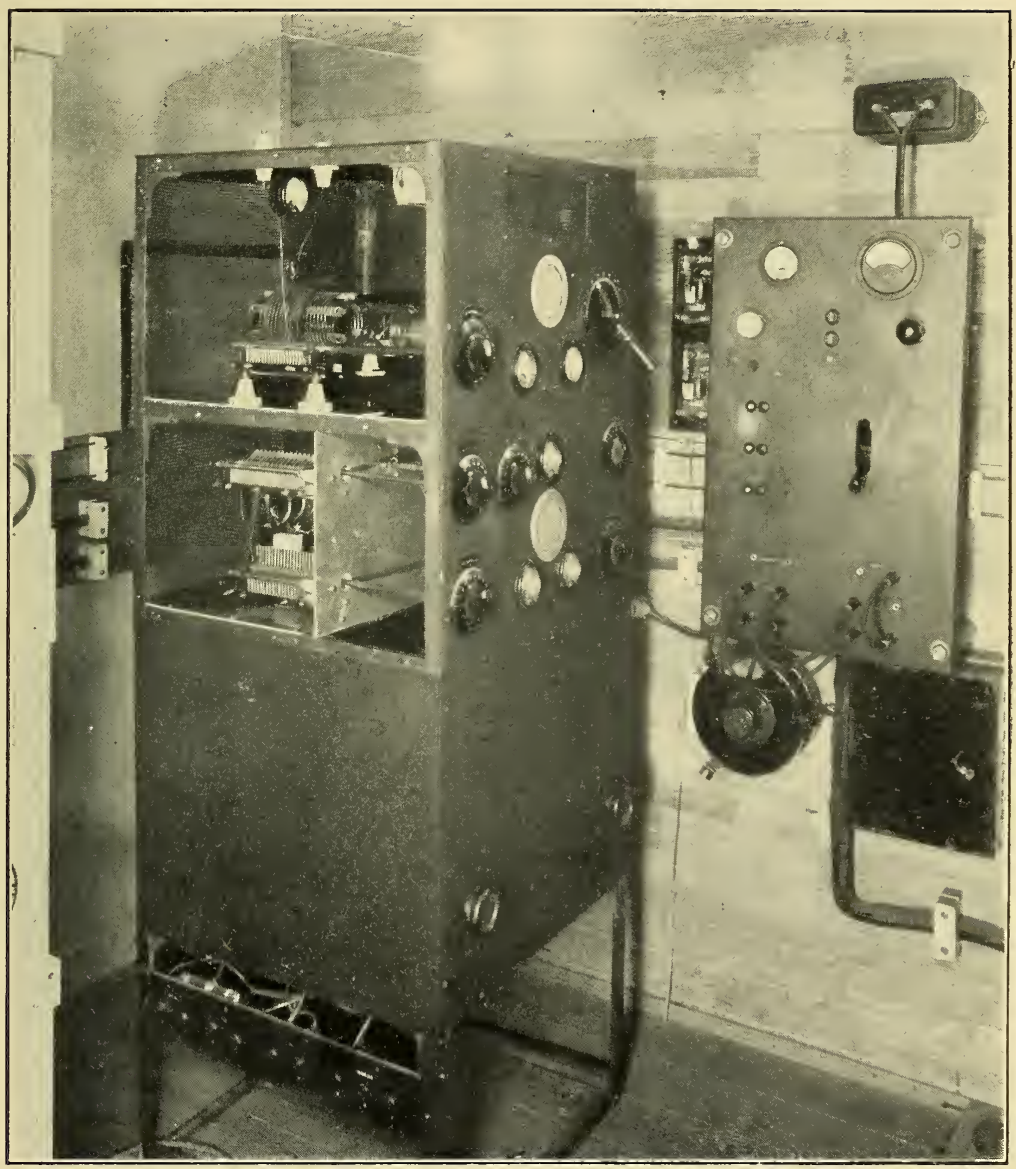

FIGURE 1.-Early standard-frequency transmitter-1,500 to $10,000 \mathrm{kc} / \mathrm{s}$. Multiplier-amplifier oscillator systems. 
B.S. Journal of Research, RP630

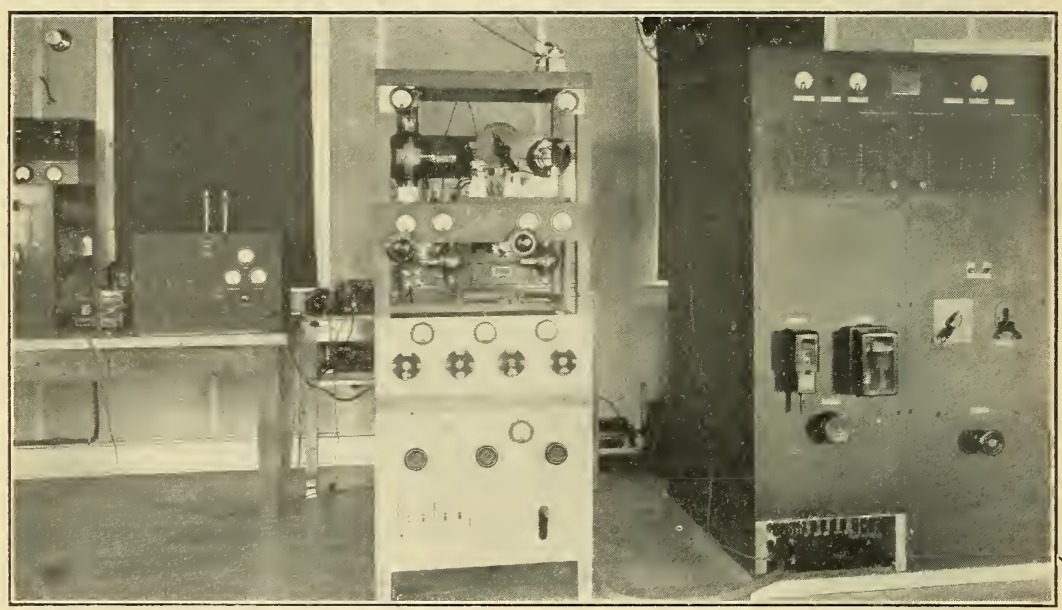

FIGURE 3.-1-kw experimental 5,000-kc/s transmitter with rectifier. 
included the reduction of the oscillator power and the installation of intermediate screen-grid amplifiers to minimize the effects of all external influences on the oscillator, the use of continuously variable inductors throughout the transmitter, and, finally, an increase in power output to provide better distance coverage. This type of transmitter was abandoned, however, in favor of equipment with more elaborate and dependable frequency control.

\section{CHANGING DEMANDS OF THE SERVICE}

The service heretofore described made a number of frequencies conveniently available through the greater part of the radio frequency spectrum. With the growth of large communication systems, there has been a development of elaborate working standards and monitoring stations whereby all radio stations in a system have their frequencies accurately checked. In addition, several commercial organizations offer a general frequency-monitoring service. Broadcasting stations are required by law to have accurate frequencymonitoring equipment. The American Radio Relay League conducts a schedule of standard-frequency transmissions which serves the amateur bands. The Federal Radio Commission maintains a number of monitoring stations for the careful checking of all station frequencies. There has thus been a change in the type of service demanded from an extensive range of standard frequencies formerly provided to a basic frequency of extreme accuracy directly controlled by the national frequency standard. Such a single frequency makes available a direct, convenient, and accurate comparison with the national standard to every radio inspector, communication company, manufacturer of frequency standards and control units, scientific laboratory, amateur, or other interested party.

\section{CHOICE OF FREQUENCY}

With this fact in mind, efforts were made to determine a suitable single radio frequency for the purpose. The advantages of a low or medium frequency were more than offset by the cost of the required installation, while broadcast frequencies were undesirable for obvious reasons. The choice, therefore, lay in the region above 1,500 kc/s. A study of transmission phenomena for day and night, winter and summer conditions showed that no single frequency would actually give universally satisfactory service. A frequency of $5,000 \mathrm{kc} / \mathrm{s}$ was chosen as the best compromise value, because of its usual lack of skip distance and yet its comparatively wide coverage, its relative freedom from interference with previously assigned stations, and its convenient integral relation with most frequency standards. The harmonic frequencies of $10,000,15,000$, and $20,000 \mathrm{kc} / \mathrm{s}$ were also chosen for future experimental purposes.

\section{SYNCHRONIZED OSCILLATORS}

The national primary standard of frequency maintained by the Bureau consists of a group of $100-$ and $200-\mathrm{kc} / \mathrm{s}$ piezo oscillators ${ }^{1}$ which are compared daily with the Naval Observatory time signals.

1 A high precision standard of frequemcy. W. A. Marrison. Proc. I.R.E., vol. 17, p. 1103, July 1929. Bell System Tech. Jour., vol. 8, p. 493, July 1929; The accuracy of the primary frequency standard of the Bureau of Standards. C. G. McIlwraith. Trans. Am. Geophysical Union, Twelfth Annual Meeting (Ntl. Acad. Sci., Washington, D.C.), p. 29, 1931. 
The direct control of the transmitted frequency by the standard presented the problem of multiplying the standard frequency of 100 or $200 \mathrm{kc} / \mathrm{s}$ to the desired $5,000 \mathrm{kc} / \mathrm{s}$. Preliminary experiments in frequency multiplication were conducted using the principles of the harmonic amplifier, the multivibrator, and the synchronized oscillator. $^{2}$ The most effective multiplication was obtained with a selfexcited oscillator operating in synchronism with a harmonic of an impressed frequency. A type '10 (7.5-watt) triode was employed in a tuned-grid oscillator circuit and the frequency from the standard was applied directly to the grid. A high value of grid-bias resistor was used and the regeneration was so adjusted that oscillations were just maintained. Correct operation was determined by a monitoring detector-amplifier loosely coupled to the oscillatory circuit. As the frequency of the latter was adjusted to approach the desired harmonic of the standard, the beat note between the two gradually decreased until, at a certain low value, it suddenly disappeared, showing that the harmonic of the standard-frequency source was now controlling the oscillator. Further adjustment of the tuning over a small range did not disturb this control. Adjustment beyond this range was indicated by the reappearance of the beat note in the telephones or loud speaker. The range of control or synchronization depended on the voltage and wave form of the input frequency, the type and relative power of the oscillator under control, and the order of multiplication. ${ }^{3}$

An examination of the operation of a synchronized oscillator was made with a cathode-ray oscillograph. One set of plates was connected to the control frequency source and the other to the oscillatory circuit. The latter was then tuned to various harmonics of the input frequency. Synchronization was indicated by a stationary figure. ${ }^{4}$ At either end of the control range a Lissajous loop figure appeared with the number of loops corresponding to the harmonic number while at the center of the range these loops collapsed into a line or repeating figure. Interpretation shows that at the center of the control range the peaks of the fundamental and harmonic voltages are coincident in time regardless of polarity. A variation of tuning through the range produces a phase shift in the harmonic voltage wave of approximately plus or minus $90^{\circ}$ or a total of $180^{\circ}$. This is independent of the order of harmonic at which the circuit is operating.

2 On some experiments in which two neighbouring maintained oscillatory circuits affect a resonating circuit. J. H. Vincent. Proc. Phys. Soc., London, vol. 22, p. 84, Feb. 15, 1920.

The automatic synchronization of triode oscillators. E. V. Appleton. Proc. Camb. Phil. Soc., vol. 21, p. 231, 1922.

Sur la synchronization harmonique des oscillations électriques. M. J. Mercier. Comptes Rendus, vol. 174 , p. 448 , Feb. $13,1922$.

De la synchronization harmonique et multiple. M. J. Mercier. Journal de Physique, vol. 5, p. 168, June 1924 .

A new method of obtaining frequency stabilization of a transmitter by means of an oscillating quartz crystal. C. W. Goyder. E. W. \& W. E., III, p. 717, December 1926.

Forced oscillations in a circuit with nonlinear resistance. B. van der Pol, Jr. Phil. Mag., vol. 3, p. 65, January 1927.

A new frequency transformer or frequency changer. I. Koga. Proc. I.R.E., vol. 15, p. 669, August 1927. Über Erzwungene Schwingungen in Elektronenröhrenkreisen. H. Witte. Arch. f. Elektrot., vol. 23, p. 84 , Nov. 5,1929 .

Le fréquencemètre étalon absolu du Laboratoire National de Radioélectricitê. B. Decau. L'Onde Électrique, vol. 9, p. 449, October 1930.

Über die Mitnahmeerscheinungen an Röhrengeneratoren Bei Verschiedenen Frequenzverhaltnissen.

H. Winter-Günther. Zeitschr. f. Hochf. Tech., vol. 37, p. 39, February 1931.

Synchronization of Westinghouse radio stations WBZ and WBZA. S. D. Gregory. Radio Engineering, vol. 11, p. 29, September 1931 .

3 See footnote 2 , above.

4 The cathode ray oscillograph. J. B. Johnson. Bell System Tech. Jour., vol. XI, p. 23, January 1932. 
A succession of synchronized oscillators was required to attain the desired frequency for transmission. The first oscillator was tuned to the fundamental of the $100-$ or $200-\mathrm{kc} / \mathrm{s}$ standard to give an ouptut rich

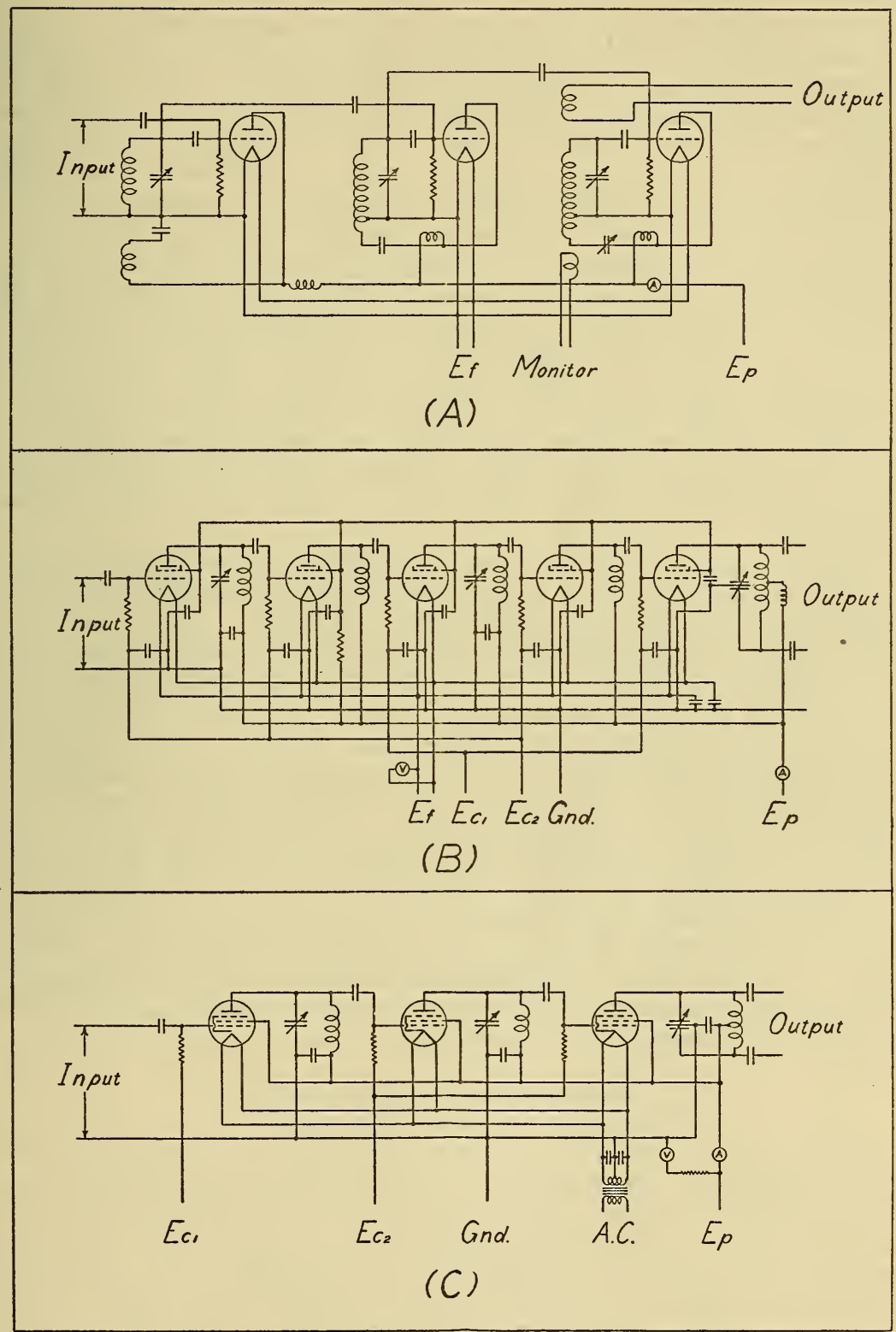

FIGURE 2.-(A) Controlled-oscillator multiplier, (B) UX-865 harmonic amplifier, (C) Pentode harmonic amplifier.

in harmonics. The second operated at $1,000 \mathrm{kc} / \mathrm{s}$ with a rich harmonic output, and, in turn, controlled a third oscillator at $5,000 \mathrm{kc} / \mathrm{s}$; figure $2(A)$. Type ' 10 tubes were used in each stage. Once they were set 
in the middle of the control ranges, as indicated by the monitor, and allowed to reach constant temperature, the system would remain in a synchronized condition until disturbed by voltage or temperature changes.

This frequency multiplier, with the addition of two stages of pushpull screen-grid power amplification, was set up as an experimental 150 -watt transmitter at the Bureau of Standards. The transmitted signals were received at a field station 8 miles away and compared with a portable secondary standard. At this point the signals were satisfactory for frequency measurement or calibration purposes. However, the transmissions caused objectionable interference with other Bureau projects. This necessitated the removal of the transmitter to the field station where it was controlled by $200-\mathrm{kc} / \mathrm{s}$ standard oscillators. ${ }^{5}$ The signals were then received and measured at the Bureau. A continuation of tests over a period of several months showed the feasibility of standard-frequency transmissions with this system. An official schedule was issued and weekly transmissions were begun in January 1931. They were continuously measured at the Bureau and the frequency of the controlling standard adjusted to the correct value in terms of the primary standard. The nominal power output was soon increased to $1 \mathrm{kw}$ and the antenna system improved.

While the results were highly encouraging, this multiplying system lacked the desired stability and required frequent monitoring by the operator to maintain control throughout all stages. If one oscillator drifted out of control because of temperature or voltage variations, a beat frequency modulation was impressed on the transmitted signal and radiation occurred on more than one frequency. ${ }^{6}$ Furthermore, in case of power failure at the input frequency the control would be lost throughout the system and the transmitter would continue to operate at reduced output on a number of frequencies corresponding to the harmonics of the oscillator stages. These difficulties are characteristic of systems capable of independent oscillation such as the controlled oscillator and the multivibrator. It is very desirable in the transmission of standard frequencies that in either of these events, however rarely they may occur, the transmitter cease to radiate. Rather than attempt to provide features for automatic readjustment or shutdown in case of loss of control, experiments with nonoscillating multiplying systems were conducted.

\section{HARMONIC AMPLIFIERS}

The harmonic amplifier principle appeared to be the only satisfactory solution to the problem. Numerous tubes were tried in various circuits with multiplication factors of 2 to 10 . Of the tubes available at the time, the type 865 (7.5-watt) screen-grid tube was found best suited to harmonic amplification. By applying slight over-excitation with a high $d-c$ and resistor-bias voltage a fair output voltage could be obtained at the fifth harmonic of the excitation frequency. The low current consumption and plate dissipation of this tube resulted in economical operation. It was necessary, however, to supply additional amplification between two such multiplying stages. An imped-

5 A 200-kilocycle piezo oscillator. E. G. Lapham. B. S., Jour. Research, vol. 11, p. 59, July 1933.

6 Stabilisateurs piezo -électriques II. J. Jammet. L'Onde Électrique, vol. 7, p. 63, February 1928. 
ance-coupled, rather than tuned, amplifier was employed. This gave ample grid swing on the succeeding tube, simplified adjustments, and added stability. Accordingly, a complete multiplier unit was built, consisting of one tuned stage at $200 \mathrm{kc} / \mathrm{s}$, an impedance-coupled stage, a tuned stage at $1,000 \mathrm{kc} / \mathrm{s}$, a second impedance-coupled stage, a tuned stage at $5,000 \mathrm{kc} / \mathrm{s}$, figure $2(B)$, followed by a push-pull buffer amplifier. The type 865 tube was used throughout. Interstage shielding was not used in the experimental model, but an external shield was employed to avoid reaction from the power amplifying stages.

The operation of this type of multiplier was in all respects similar to that of the usual frequency doubler. Neither supply voltages nor tuning adjustments were found to be critical. A high degree of stability was obtained which was unaffected by any reasonable variation in temperature or voltage or mechanical disturbance. An oscillographic study revealed a phase shift with tuning, similar to that noted in the synchronized oscillator. If such a shift were induced in a low-frequency stage by a variation of the tuning control, it would be multiplied by the succeeding stages and would result in a larger phase shift in the output frequency, amounting to a net gain or loss of several complete cycles. All changes in tuning, however, are kept to a minimum during a scheduled transmission to insure the stability of the output frequency.

The main object of the experiments, as stated before, was therefore achieved. In the event of failure of any part of the equipment or input frequency, the multiplier ceased to function and the transmitter could not radiate. There was no source of radio-frequency voltage other than the standard, the entire transmitter operating only as an amplifier.

While designed for a single-frequency output, this frequency multiplier offered unusual flexibility. With a $200-\mathrm{kc} / \mathrm{s}$ input frequency the first multiplying stage could be set at any harmonic in its tuning range from 600 to $1,800 \mathrm{kc} / \mathrm{s}$. Corresponding harmonics of these frequencies could be obtained in the second multiplying stage as high as $8,000 \mathrm{kc} / \mathrm{s}$. It is easily seen that by the addition of the usual doubling or tripling amplifiers a wide variety of frequencies covering the high-frequency spectrum was available.

The choice of tubes and circuit operating conditions for an efficient harmonic amplifier has been the subject of much study and experimentation. Marique ${ }^{7}$ made an algebraic analysis of the operation of triodes as frequency multipliers. He showed that it is desirable to use tubes of high mutual conductance and low internal resistance. Of the low-power American tubes having these characteristics, the best examples were the types ' 50 , ' 45 , and ' 10 , in the order named. This analysis did not assume positive grid swing or saturation plate current of the tube, both of which were present in the frequency multipliers used. The screen-grid type 865, for instance, was found to have marked advantages over any of the triodes above mentioned, although it had neither of the characteristics advocated. It is evident that a high amplification factor is also advantageous. The advantages of this tube lay in the greater amplitude of harmonics, smaller amplitude of fundamental component due to low interelectrode capacity, and less critical bias voltage required.

${ }^{7}$ Note sur le Calcul des Étages Multiplicateurs de Frêquence à Triodes. J. Marique. L'Onde Électrique, vol. 8, p. 1, January 1929. 
The most promising tube for this purpose was the pentode type which had in addition to a screen grid, a suppressor grid for the elimination of secondary emission from the plate. This tube, had all the desirable characteristics mentioned above, namely, a high mutual conductance and amplification factor and a relatively low internal resistance. A harmonic amplifier was built at the Bureau of Standards using the type 247 pentode with unusually good results; figure $2(C)$. A multiplication of 5 to 1 was obtained in one tube with a slight voltage gain. No intermediate amplification was required. Three tubes were sufficient to give a peak output of more than 100 volts at $5,000 \mathrm{kc} / \mathrm{s}$ from an input of less than 5 volts at $200 \mathrm{kc} / \mathrm{s}$. This unit was installed and given thorough tests in an auxiliary transmitter before incorporation in the 1-kw transmitter used in the scheduled transmissions. These tests showed the type 247 multiplier to be fully as stable and to have a lower noise level than the type 865 multiplier, while the tube and operating costs were much reduced. As in the previous multiplier units, a type 865 push-pull buffer amplifier was used.

In the experimental 1-kw transmitter, the multiplier unit with buffer amplifier was followed by two stages of push-pull intermediate type 860 (150-watt) amplification and a push-pull type 861 (1-kw) power amplifier. The latter was coupled through a transmission line to a horizontal half-wave antenna approximately one eighth wave length above ground. An antenna of greater height was not permissible because of the proximity of an experimental flying field. The equipment was in weekly operation from March 1931 to November 1932, at which time a new transmitting station was completed at Beltsville, Md., about 13 miles from the main Bureau laboratory. The experimental transmitter was then moved to the new location, a new rectifier-type power supply provided, and higher antennas constructed. This transmitter, together with the high-voltage rectifier and control-frequency standard, are shown in figure 3.

\section{30-KW TRANSMITTER}

A great step in the progress of the standard-frequency service was marked by the purchase and installation, late in 1932, of a high-power transmitter of the most advanced design. It has a continuous frequency range of 4,000 to $26,000 \mathrm{kc} / \mathrm{s}$ and a nominal power output of $30 \mathrm{kw}$ at the lower frequencies. It consists of a 1-kw exciter unit, with its associated rectifier power supply, a $30-\mathrm{kw}$ power amplifier, main-plate rectifier, plate transformer, tap-changing contactors, and a water-cooling system for the large transmitting tubes. A block diagram of the equipment is given in figure 4 , while a simplified schematic diagram is shown in figure 7. Photographs of the major units of the installation at the Beltsville field station are shown in figures 5 and 6.

The exciter unit, together with its rectifier, is a complete and independent $1-\mathrm{kw}$ transmitter. It contains a 7.5 -w type 865 oscillator or buffer amplifier, $B$, figure 4 , four intermediate type 860 screengrid, fundamental, doubling, or tripling amplifiers $(C),(D),\left(E^{\prime}\right),(F)$, and a power amplifier using two type 861 (500-w) screen-grid tubes in parallel $(G)$. A frequency multiplier, as shown in figure $2(C)$ and at $A$ in figure 4 , is built as an integral part of the unit. With a 
B.S. Journal of Research, RP630

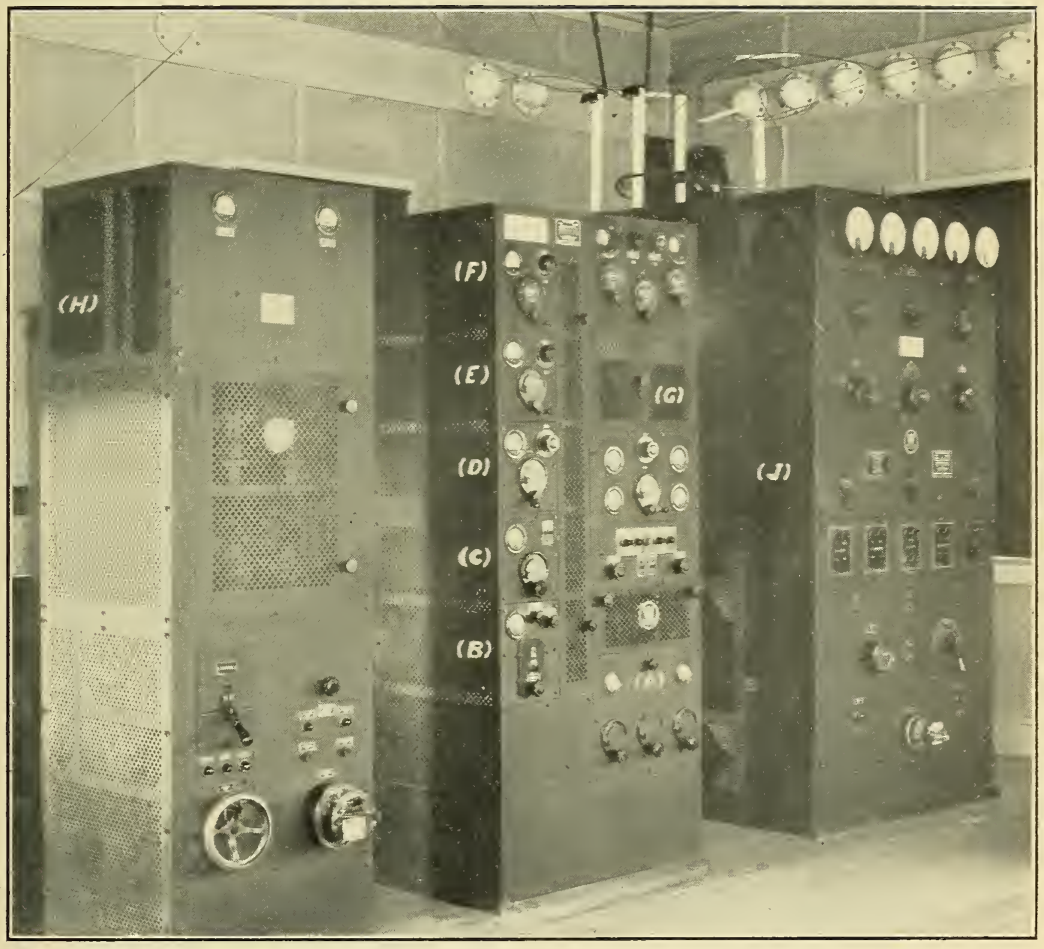

FiguRe 5.-30-kw transmitter units on main floor of Beltsville, Md., station. 
B.S. Journal of Research, RP630

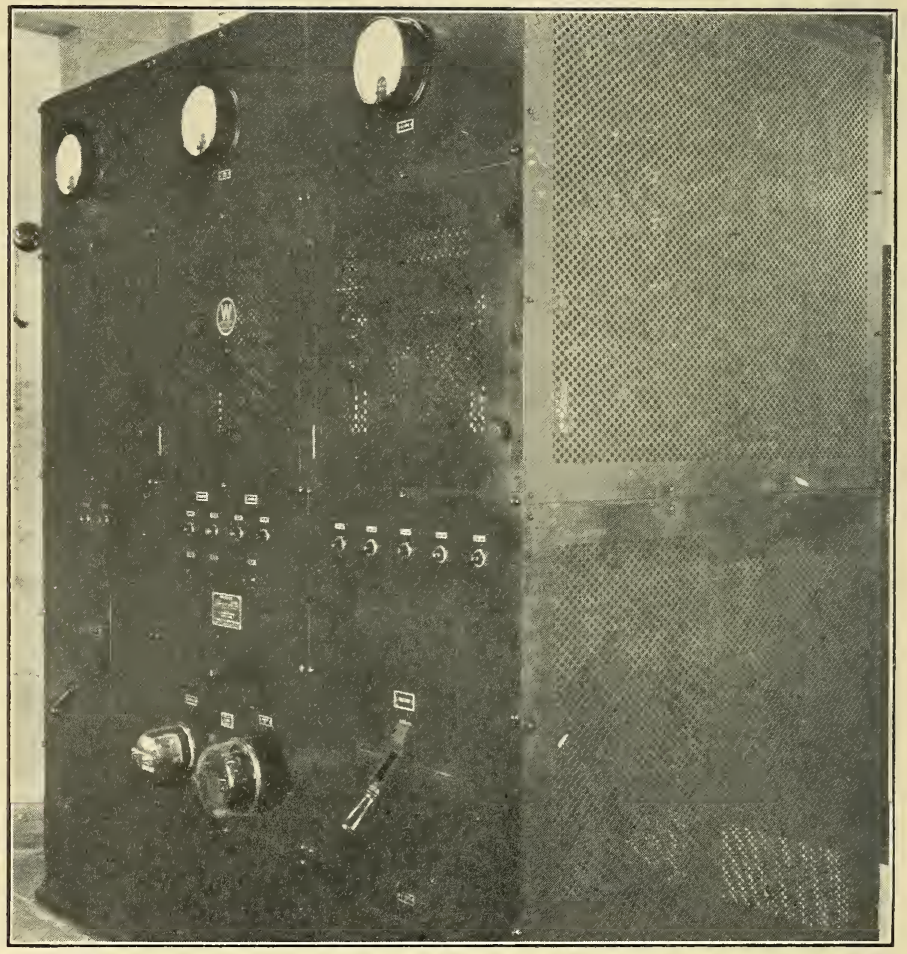

FigURE 6.-30-kw transmitter units in basement of Beltsville, Md., station. 
$200-\mathrm{kc} / \mathrm{s}$ input from a standard, this transmitter will deliver from 600 to 1,200 watts to any type of antenna or to the power amplifier grids at $5,000 \mathrm{kc} / \mathrm{s}$ or any harmonic up to $20,000 \mathrm{kc} / \mathrm{s}$. With the type 865 $(7.5-\mathrm{w})$ tube used as a self-excited oscillator, the unit will operate in a similar manner on any frequency from 4,000 to $26,000 \mathrm{kc} / \mathrm{s}$.

The power-amplifier unit, figure $4(J)$, employs two type 858 $(20-\mathrm{kw})$ water-cooled tubes in a push-pull, tuned-grid, tuned-plate circuit arrangement. Coupling to the antenna is effected through $600-\mathrm{ohm}$ matched-impedance transmission lines. The frequency is continuously variable from 4,000 to $26,000 \mathrm{kc} / \mathrm{s}$ by controls on the front panel of the unit. The plate inductors and associated switches

(F)

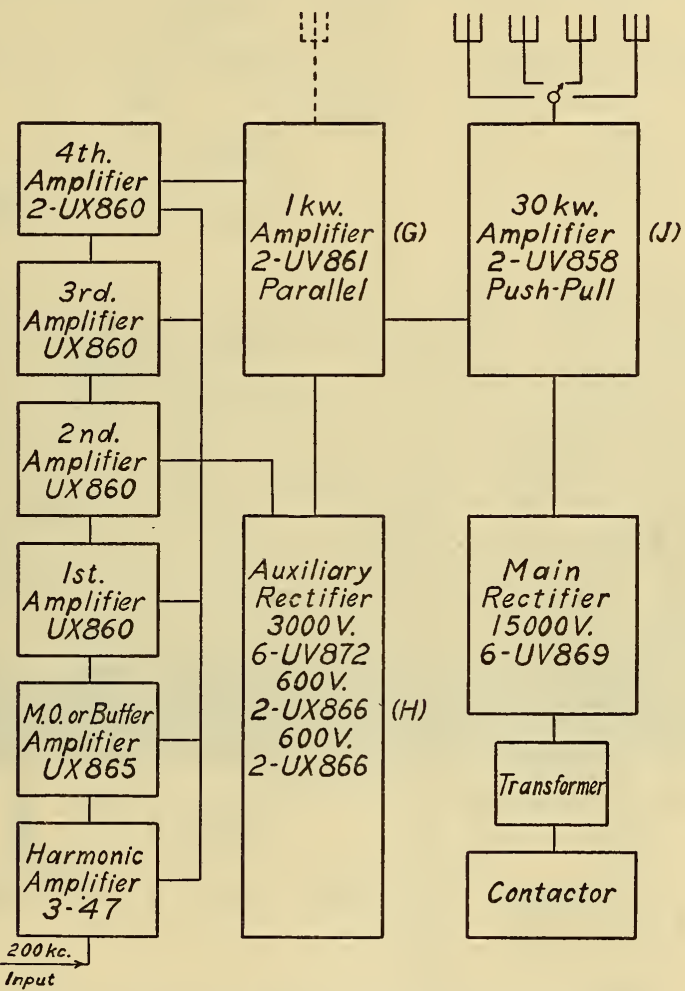

FrguRe 4.-Block diagram of 30-kilowatt transmitter

as well as the plates of the tubes are cooled by circulating distilled water. Complete control of all starting operations, plate voltages, and frequency adjustment is obtained through switches and dials on the power amplifier and exciter front panels. This affords great flexibility and convenience in operation.

All plate and bias voltages are obtained from rectifiers using hot-cathode mercury-vapor tubes. The power-amplifier plate voltage of 7,000 to 15,000 volts is supplied by the main rectifier equipment, using six type 869 tubes in a full-wave three-phase circuit. The exciter plate voltages of 1,500 and 3,000 volts and the power-amplifier bias voltage are supplied from rectifiers using type 872 tubes, while lower-plate and bias voltages are supplied from type 866 tubes. The 


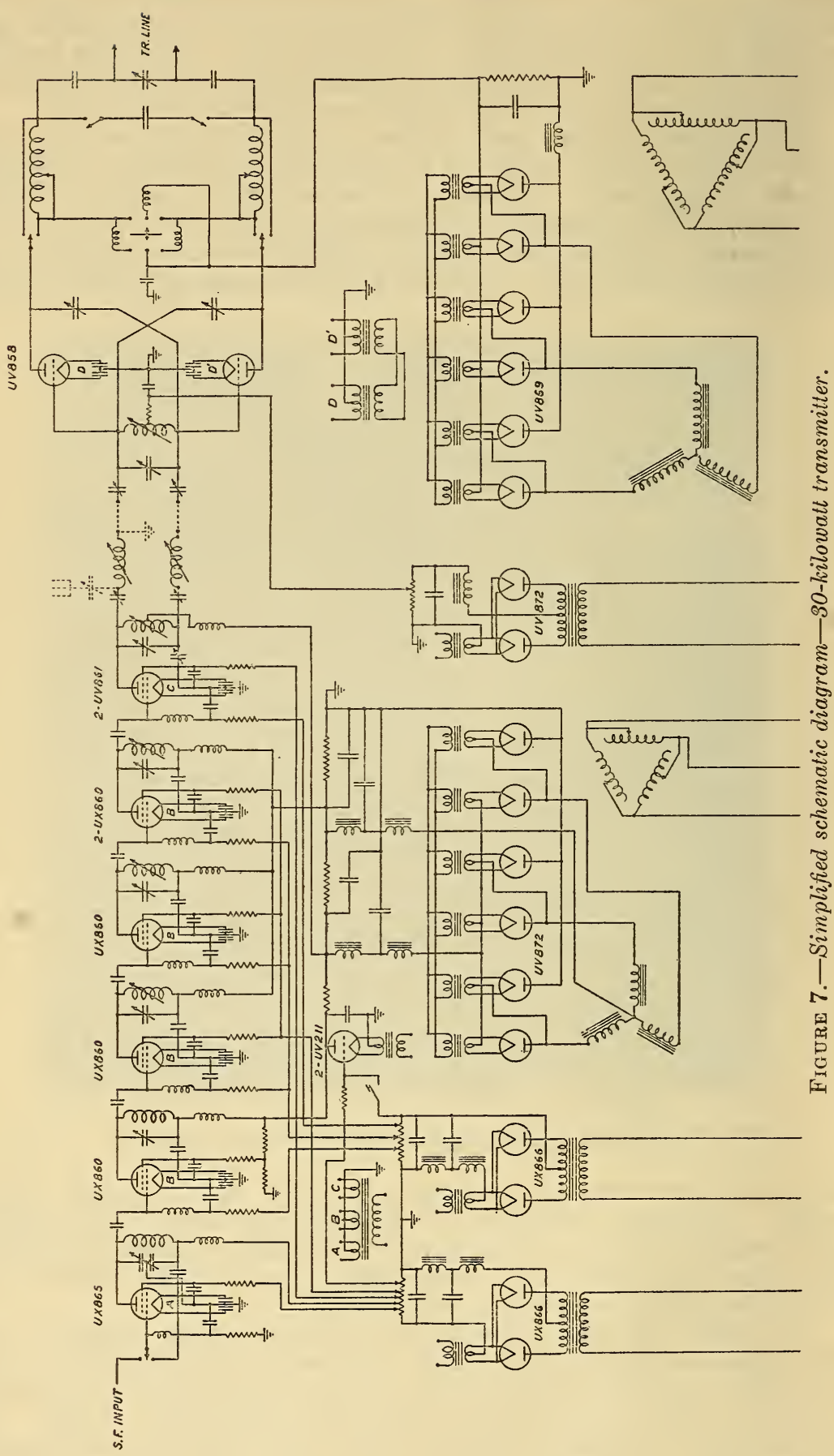


B.S. Journal of Research, RP630

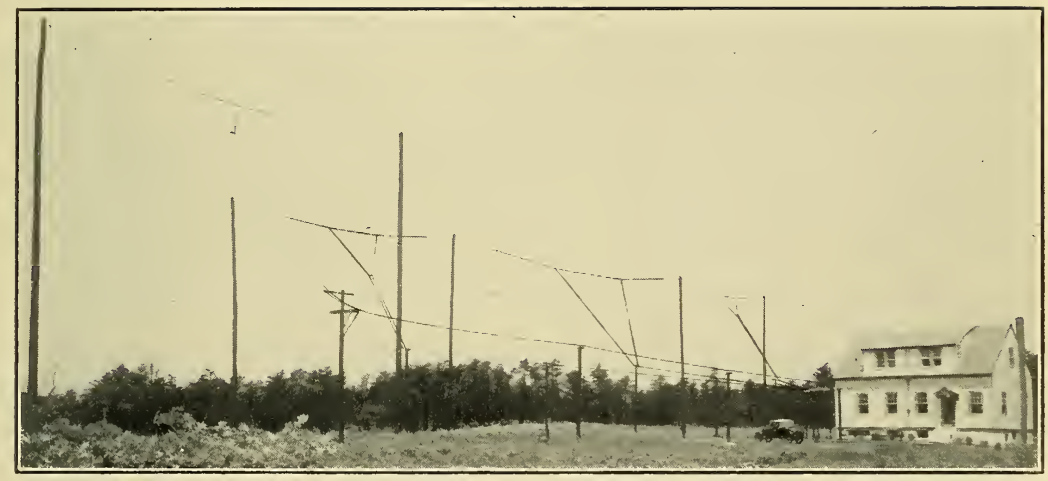

Figure 8.-View of antennas and transmitting station building at Beltsville, Md. 
main plate voltage is adjustable by means of large contactors which change the taps on the primary of the high-voltage three-phase transformer. A similar, but less elaborate system, is used to regulate the exciter plate voltages.

The water-cooling system for the power amplifier consists of two large radiators with associated fans, a centrifugal water pump, and a storage tank for distilled water. All connections between units are of brass pipe to reduce corrosion and resulting increase in the water conductivity.

An elaborate interlocking system is provided between all units to effect immediate shut-down of the transmitter in case of excess plate current, low filament voltages, excess water temperature, low water pressure or flow, or the opening of any access door in the equipment. This insures protection to tubes and personnel in case of improper operation or inadvertent entrance to any compartment for inspection or adjustment while operating voltages are on.

The limitation of the transmitted frequencies to $5,000 \mathrm{kc} / \mathrm{s}$ and harmonics thereof made the use of fixed-frequency antennas advantageous, especially in view of the use of matched-impedance transmission lines to the power amplifier. A single-wire horizontal half-wave doublet was therefore installed for each operating frequency, $5,000,10,000,15,000$, and $20,000 \mathrm{kc} / \mathrm{s}$. The antennas were suspended from wood poles $65 \mathrm{ft}$ in height. This gave each antenna a height much more than a quarter wave length above ground and a substantial amount of low-angle radiation. They were oriented in a north-south direction so that the slight directional characteristics favored the western part of the United States. A view of the station building and antenna system is shown in figure 8 .

\section{RESULTS}

Since the inauguration of the $5,000-\mathrm{kc} / \mathrm{s}$ transmissions in January 1931 , there has been a progressive increase in accuracy of the transmitted frequency. In order to maintain the frequency continuously in agreement with the national primary frequency standard, the signals are received and the frequency continuously recorded at the main laboratory of the Bureau of Standards, 13 miles distant. Adjustments of the control standard in the transmitting station to agreement with the national primary standard are made just prior to each scheduled transmission. The constancy of this control standard is such that this agreement is usually maintained over a 2-hr schedule without any readjustment being required. The average deviations of the transmitted frequency from the national standard are about two parts in a hundred million, while extreme variations may reach five parts in a hundred million.

Because of phenomena which may occur in the transmitting medium the frequency as received at some distant points may sometimes not have this accuracy. ${ }^{8}$ Apparent frequency changes greater than a part in 10 million are sometimes noted at a distance of 13 miles when there is ample evidence that they are not present in the transmitted wave.

\footnotetext{
${ }^{8}$ Radio dissemination of the national standard of frequency. J. H. Dellinger and E. L. Hall. Radio Engineering, vol. 12, p. 23, May 1932.
} 
The 30-kw transmitter has been used regularly for the standard frequency schedule since April 1933. The increase in power has resulted in marked improvement in the dependability of the signals, especially in those areas where reception is ordinarily difficult. Reports of reception have come from every section of the United States as well as points in Alaska, the Canal Zone, England, and Italy. In localities where receiving conditions are at all favorable observers report successful use of the signals for frequency comparison purposes.

Washington, August 26, 1933. 